\title{
Comparative Evaluations of Mass Spectral Databases
}

\author{
Stephen E. Stein, Pierre Ausloos, and Sharon G. Lias \\ Chemical Kinetics and Thermodynamics Division, National Institute of Standards and Technology, \\ Gaithersburg, Maryland, USA
}

This Communication presents a statistical analysis of the distributions of the National Institute of Standards and Technology/Environmental Protection Agency/Mass Spectrometry Data Center Mass Spectral Database and the Wiley Registry of Mass Spectra according to sizes (peaks per spectrum) of spectra. The differences in the distributions are explained in terms of the different philosophies under which the two databases were built. The Wiley Registry is a collection that attempts to include all available spectra, including spectra of unique compounds derived from the literature. The NIST collection selects primarily spectra of interest for chemical analysis. (/ Am Soc Mass Spectrom 1991, 2, 441-443)

$\mathrm{M}$ cLafferty et al., in an accompanying communication [1], discuss various aspects of the distributions by size of the Wiley Registry of Mass Spectra [2] and the database maintained and distributed by the National Institute of Standards and Technology (NIST) [3]. These two large databases of mass spectra originated from a common set of older mass spectral databases, and therefore have 36,847 spectra in common. (These spectra are not necessarily identical in the two databases because of different error analysis and editing of the spectra.) During the past 20 years these two widely used databases developed in different directions and the contents of the collections diverged. The NIST database reached its present size of 53,994 spectra at a relatively slow rate; much of the increase in the size has come about through directed determinations of original spectra. The Environmental Protection Agency (EPA), for example, commissioned the determination of several thousand spectra to ensure that the database contained spectra for selected compounds of special environmental interest. The database is conceived solely as a tool for the analytical chemistry community, and does not attempt to include all available mass spectra.

The Wiley database, on the other hand, has grown to include 139,859 spectra under a policy in which all available spectral information is included, including spectra for isotopically labeled substances, partial spectra of newly synthesized compounds, and multiple spectra of a single compound. Largely because of the common origin of the two databases, and because of a licensing agreement under which the Wiley Registry includes spectra from the NIST mass spectral

Address reprint requests to Sharon G. Lias, National Institute of Standards and Technology, Gaithersburg. MD 20899. database that are not in the Wiley collection, the 5th edition of the Wiley Registry contains 48,725 spectra in common with the NIST collection $190.2 \%$ of the spectra in the distributed NIST database), although many of these spectra are not alike in the two databases because of editing by Wiley.

Because of these different approaches to building the databases, the statistical distribution of spectra is very different in the two databases. In this Communication we report on a comparative statistical analysis of the 5th edition of the Wiley Registry of Mass Spectra and the 1990 release of the NIST/EPA/MSDC Mass Spectral Database, and discuss some of the implications for library searching.

The percentages of spectra in the two collections having a given number of peaks are shown in Figure 1. In Figure 1a, two distribution curves are shown for the Wiley collection: one set represents the database as currently distributed, with spectra to which computer-generated isotopic peaks have been added, and the other represents a curve for the same database without those added peaks. (In the Wiley Registry isotopes are computed for the parent ion and predictable fragment ions. In the case of more complete spectra, where the computer-added peaks often replace the originally reported abundances, the impact on the number of peaks is minimal, but for the 17,000 spectra that originally had between one and five peaks, the size of the spectrum is typically more than doubled.) In the discussion that follows, we will consider only the distributions without added isotopic peaks because this allows a direct comparison of the size distributions of the Wiley and the NIST collections. Striking differences in the distributions by size of spectra in the two databases are shown in Figure 1b. The Wiley Registry data show a broad and a sharp 

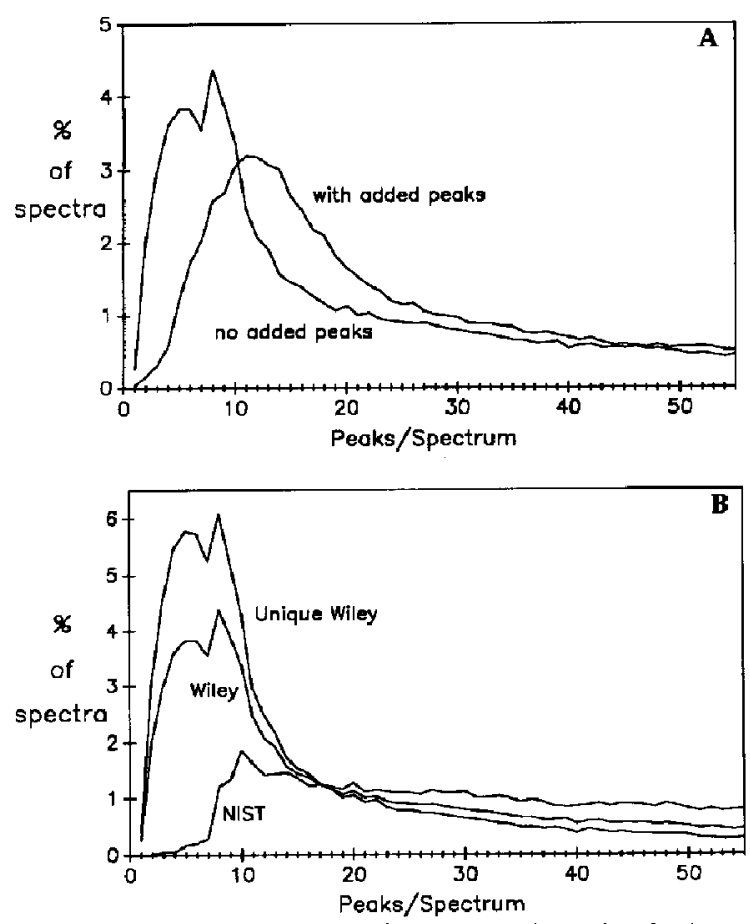

Figure 1. (a) The percentage of spectra in the Wiley Registry. 5th edition, having a given number of peaks. The two curves represent the database as currently distributed with isotopic peaks added to the spectra, and the same collection of spectra without the computer-added isotope peaks. (b) The percentage of spectra having a given number of peaks for the 1990 version of the NIST database, the Wiley Registry. 5th edition, and the collection of spectra that is unique to the Wiley database (i.e., without the presence of spectra from the NIST database). In (b), the collections of spectra are placed on the same hasis by comparing databases for which spectra have been treated the same, i.e., without added isotope peaks.

maximum at the low end of the distribution curve. The broad maximum, near 5 and 6 peaks /spectrum, is typical of the size of spectra abstracted from journal articles, while the sharp maximum, at 8 peaks $/$ spectrum, originates from the inclusion of 3,692 Imperial Chemical Industries spectra that are part of the Eight Peak Index [4].

Statistics gleaned from these distributions (Table 1) clearly demonstrate the effects of the policies under which the databases were built. Although, as pointed out by McLafferty et al. [1], the number of peaks most commonly found in the NIST/EPA/MSDC Database is 10 , while that in the Wiley collection is 11 (including computer-added isotopic peaks) or eight (unaltered spectra), these numbers do not reveal the striking differences in the structures of the two databases. For example (Table 1), if we examine the spectra unique to the Wiley collection, we find that this subset has $41.6 \%$ spectra with fewer than 10 peaks, as compared to $3.5 \%$ in the NIST database. The median size of a NIST spectrum is approximately twice as large as that of a spectrum from the Wiley collection, and nearly five times as large when one considers the subset of spectra unique to the Wiley collection. (The median sizes of spectra-50\% of spectra are larger and $50 \%$ are smaller - are more instructive in revealing the structure of a database than are average spectral sizes. This is so because of the uneven distribution of spectral sizes.)

Some 61,342 spectra from the Wiley collection were derived from 13 different journals, and have a median spectrum size of 9.0 peaks per spectrum; the NIST database has 6,724 of these same spectra with a median spectrum size of 27.8 peaks per spectrum*. The fact that spectra derived from journal articles are, on the whole, more complete in the NIST database than in the Wiley collection can be explained; in the NIST database, the collection of spectra from the literature is done under a protocol that requires the original author to donate the complete verified spectrum to the database. A few incomplete spectra that have been included in the past are gradually being removed as more complete versions are obtained. There are two reasons for this policy requiring the inclusion of complete spectra only: (1) the lower desirability of incomplete spectra for use in library searching is generally recognized, for instance, by the quality index algorithms developed by McLafferty and co-workers [5] (see Table 1) and by Terwilliger et al. [6], which penalize spectra having fewer peaks than one would predict from the number of atoms in the molecule; and (2) as mentioned above, many incomplete spectra that are available are spectra of compounds of limited general interest for analytical chemistry.

As has been emphasized by McLafferty et al. [2], the inclusion of replicate spectra (different spectra for the same molecule) improves the matching statistics in library searching. The NIST library has not in the past included replicates; instead, only a single spectrum of a given compound - that having the highest computer-generated "quality index" - has been provided. Many of the replicates in the NIST spectral archive were of low quality, and hence of questionable value. However, during the past year all of the replicate spectra in the NIST archive of spectra have been individually examined by mass spectrometrists,

*I. Org, Chem.: Wiley, 9993 spectra, 5.7 pk median; NIST, 262 spectra, 27.5 pk median; Helvet. Chem. Acta: Wiley, 8381 spectra, 10.0 pk median; NIST, 845 spectra, 17.0 pk median; Org. Mass Spec.: Wiley, 7187 spectra, 22.0 pk median; NIST, 3221 spectra, 33.0 pk median: Chem. Ber.: Wiley, 6131 spectra, 5.6 pk ntedian; NIST, 284 spectra, 16.5 pk median; J. Chem. Soc. (C): Wiley, 5220 spectra, 5.6 pk median; NIST, 239 spectra, 15.5 pk median; Tetrahedron: Wiley, 5182 spectra, 7.5 pk median; NIST, 358 spectra, $>40$ pk median; I. Am. Chem. Soc: Wiley, 4821 spectra, $6.5 \mathrm{pk}$ median; NIST, 579 spectra, 25.0 pk median; Aust. J. Chem.: Wiley, 4626 spectra, 8.0 pk median; NIST, 322 spectra, 24.5 pk median; I. Het. Chem.: Wiley, 2756 spectra, 6.5 pk median; NIST, 275 spectra, 19.5 pk median; Biomed. Mass Spec.: Wiley, 2893 spectra, 13.1 pk median; NIST, 100 spectra, 16.5 pk median; Can. J. Chem.: Wiley, 1891 spectra, 6.5 pk median; NIST, 147 spectra, 32.0 pk median; Acta Chem. Scand.: Wiley, 1413 spectra, 8.0 pk median; NIST, 20 spectra, $10.0 \mathrm{pk}$ median; Bull. Chem. Soc, France: Wiley, 938 spectra, 6.8 pk median; NIST, 72 spectra, $28.0 \mathrm{pk}$ av. 
Table 1. Statistical comparison of numbers of peaks in spectra of mass spectral databases

\begin{tabular}{|c|c|c|c|c|c|}
\hline & \multicolumn{2}{|c|}{ Numbers of peaks in spectrum } & \multicolumn{2}{|c|}{ Percent of spectra } & \multirow{2}{*}{$\begin{array}{l}\text { Quality index } \\
\text { Median }\end{array}$} \\
\hline & Median $^{\mathrm{b}}$ & Average & $<10$ Peaks & $>100$ Peaks & \\
\hline NIST & 52.4 & 72.2 & $\mathbf{3 . 5}$ & 22.7 & 670 \\
\hline \multirow[t]{2}{*}{ Wiley } & 21.8 & 48.0 & 28.4 & 14.2 & 340 \\
\hline & $(27.5)^{c}$ & $(53.5)^{c}$ & $(11.3)^{c}$ & $(15.5)^{\mathrm{C}}$ & \\
\hline \multirow[t]{2}{*}{ Wiley minus NIST } & 11.5 & 34.8 & 41.6 & 9.5 & 160 \\
\hline & $(17.7)^{c}$ & $(40.0)^{c}$ & $(17.0)^{6}$ & $(10.4)^{\circ}$ & \\
\hline
\end{tabular}

a Using the quality index devised by McLafferty at al. [5].

${ }^{b} 50 \%$ of spectra have fewer than this number of peaks.

Wiley spectra including computer-added isotope peaks.

dThe Wiley collection, not including any spectra from the NIST database.

who manually selected the best spectra and deleted poor quality spectra. (A complete discussion of our evaluation effort, a description of the new protocols under which the database is maintained and edited, and pertinent statistics concerning retrieval of data are being prepared for publication.) This effort is expected to improve the matching statistics of the NIST database relative to the Wiley database, since the compounds for which replicates are available are often common compounds that are of interest to analytical chemists. The next release of the NIST database (September 1991) will include an auxiliary file containing all of the remaining high quality evaluated replicate NIST spectra. The inclusion of numerous irrelevant partial spectra in a database will harm library searching

In summary, the Wiley Registry and NIST databases have developed with quite different objectives that are clearly reflected in the distributions of sizes of spectra. The Wiley database is a general purpose collection that is valuable because it contains a large fraction of all available mass spectra. The NIST database is made up primarily of complete spectra, many of which have been obtained for the specific purpose of serving as reference spectra for library searching. The statistics presented in this Communication clearly support the statement made in an advertisement that the NIST database "is the only large collection of mass spectra consisting almost entirely of complete spectra" [7].

\section{References}

1. McLafferty, F. W.; Stauffer, D. B.; Loh, S. Y. J. Am. Soc. Mass Spectrom. 1991, 2, 438-440.

2. McLafferty, F. W.; Stauffer, D. B.; Twiss-Brooks, A. B.; Loh. S. Y. J. Am. Soc. Mass Spectrom. 1991, 2, 432-437.

3. NIST/EPA/MSDC Mass Spectral Database, Standard Reference Database 1, April, 1990.

4. The Eight Peak Index of Mass Spectra, 3rd ed.; Mass Spectrometry Data Centre: England, 1983.

5. Speck, D. D.; Venkataraghavan, R.; McLafferty, F. W. Org. Mass Spectrom. 1978, 13, 209. 